\title{
Microvascular autologous submandibular gland transplantation in severe cases of keratoconjunctivitis sicca
}

\author{
Jia-Zeng Su, Zhi-Gang Cai and Guang-Yan Yu*
}

\begin{abstract}
Dry eye syndrome is a relatively common disease of the tears and ocular surfaces that results in discomfort, visual disturbance, and tear film instability with possible damage to the ocular surfaces. Microvascular submandibular gland (SMG) transfer offers a surgical alternative for a permanent autologous substitution of tears using the basal secretion of a transplanted SMG. Long-term follow-up reveals that this technique is a lasting and effective solution for patients with severe dry eye syndrome. The uncomfortable symptoms were relieved, and the frequency of use of pharmaceutical tear substitutes was reduced. Objective examination showed significant improvement in tear film and some features of ocular surface such as breakup time of tear film and corneal staining. Patients may suffer from obstruction of Wharton's duct or epiphora after surgery. Activation of secretion-related receptors could improve the early hypofunction of the denervated SMG and prevent the duct obstruction. Reduction surgery, partial SMG transplantation, uses of atropine gel or Botulinum toxin A could be the choices of treatment for epiphora.
\end{abstract}

Keywords: Obstructive sialadenitis; Keratoconjunctivitis sicca; Submandibular gland transplantation

\section{Introduction}

Keratoconjunctivitis sicca (KCS), or dry eye syndrom, is a multifactorial disease of the tears and ocular surfaces that results in discomfort, visual disturbance, and tear film instability with possible damage to the ocular surfaces [1]. The current options for treatment include artificial tear substitutes, cyclosporine eye drops, and occlusion of tear drainage [2,3]. However, these treatments have only satisfactory results in mild cases. For severe cases of KCS, none of the routine methods of symptom management are satisfactory [4].

Microvascular submandibular gland (SMG) transfer with implantation of Wharton's duct into the upper conjunctival fornix offers a surgical alternative for a permanent autologous substitution of tears using the basal secretion of a transplanted and revascularized submandibular gland. This procedure was first described by Murube-Del-Castillo in 1986 [5]. His surgical attempt was followed by MacLeod [6], Kumar [7], Geerling [4],

\footnotetext{
*Correspondence: czg4209@126.com; gyyu@263.com Department of Oral and Maxillofacial Surgery, Peking University School and Hospital of Stomatology, No. 22, South Avenue Zhongguancun, Haidian District, Beijing 100081, P.R. China
}

Sieg [8], Jia [9], Yu [10], and Paniello [11]. The reports from these groups confirmed that the secretions from all viable transplanted SMGs maintained stable function in the long term and had been effective for severe cases of KCS [12-17].

\section{Review \\ Preoperative evaluation}

Before surgery, a detailed medical history questionnaire regarding etiology, course and past treatment was taken to assess the dry eye symptoms subjectively. Detail and objective assessments, including ophthalmologic evaluation, oral and maxillofacial evaluation and scintigraphy with ${ }^{99 \mathrm{~m}}$ Tc-pertechnetate were performed. The ophthalmologic evaluation included best-corrected visual acuity, Schirmer test, break-up time (BUT), and fluorescence staining of the cornea and conjunctiva. Oral and maxillofacial evaluation included wetting of the oral mucosa, saliva pooling at the floor of the mouth, salivary secretion at the ductal orifices of parotid and submandibular glands. Scientigraphy with ${ }^{99 \mathrm{~m}}$ Tc-pertechnetate was carried out to test the secretion function of submandibular and parotid glands. Generally speaking, the SMG with a 
relative hypofunction was the first choice of donor gland $[10,18,19]$.

The indications for the surgical procedure were that the patients had persisting pronounced symptoms of dry eye, that where other previous ophthalmologic treatments had failed, and that in the ophthalmologic evaluation, Schirmer test showed a value of $<2 \mathrm{~mm}$, the BUT a value of $<5 \mathrm{sec}$, and that the cornea showed positive fluorescence staining. Contraindications were Sjőgren's syndrome, obvious symptoms of xerostomia or that the whole saliva flow rate was $<0.3 \mathrm{~g} / \mathrm{min}$, and that scintigraphy showed a severe dysfunction of multiple major salivary glands [18].

\section{Surgical procedure}

Under general anaesthesia, a curved incision was made in the temporal region (Figure 1A). An enlarged, caudally based temporal flap was retracted from the layer superficial to the superficial temporal vessels. Then the superficial vessels were carefully dissected (Figure 1B).

The submandibular gland, including Wharton's duct and its related blood vessels, was harvested from the submandibular triangle through a conventional cervical approach. The dissection of the submandibular gland was taken using an extra-capsular approach to preserve the structures of the gland. The lower part of anterior facial vein, the proximal part of facial artery and its accompanying vein were carefully dissected and protected (Figure 1C). The chorda tympani supplying the submandibular gland was severed, whereas the lingual nerve was carefully protected.
The second part of the operation was performed intraorally. A blunt probe was inserted into the duct through its orifice to label Wharton's duct. The mucosa was incised between the floor of the mouth and the base of the tongue, with a $5 \mathrm{~cm}$ length incision. A cuff of mucosa was left intact around the duct opening (Figure 1D). The duct was dissected and the lingual nerve was protected. The salivary duct of the sublingual gland which joins Wharton's duct was cut and ligated. The entire Wharton's duct was pulled out through the cervical submandibular access. Now the submandibular gland was released. Only the facial artery and lower part of anterior facial vein were left, connecting the submandibular gland to the body. The anterior facial vein was cut near the point joining itself with the common facial vein. Bleeding from the anterior facial vein which attached to the submandibular gland was observed to evaluate the state of venous drainage from the gland (Figure 1E). The proximal part of facial artery and its accompanying vein were cut at the roots of their branches from the external carotid artery. The submandibular gland was removed together with the facial vessels and Wharton's duct (Figure 1F).

Heparinized saline was irrigated into the facial artery. The seepage of the solution from the anterior facial veins, accompanying vein of facial artery and the vein close to the duct was observed. The vein with most seepage of solution was taken as the first choice for anastomosis.

Afterwards, the free submandibular gland was transferred to the temporal region. The superficial temporal

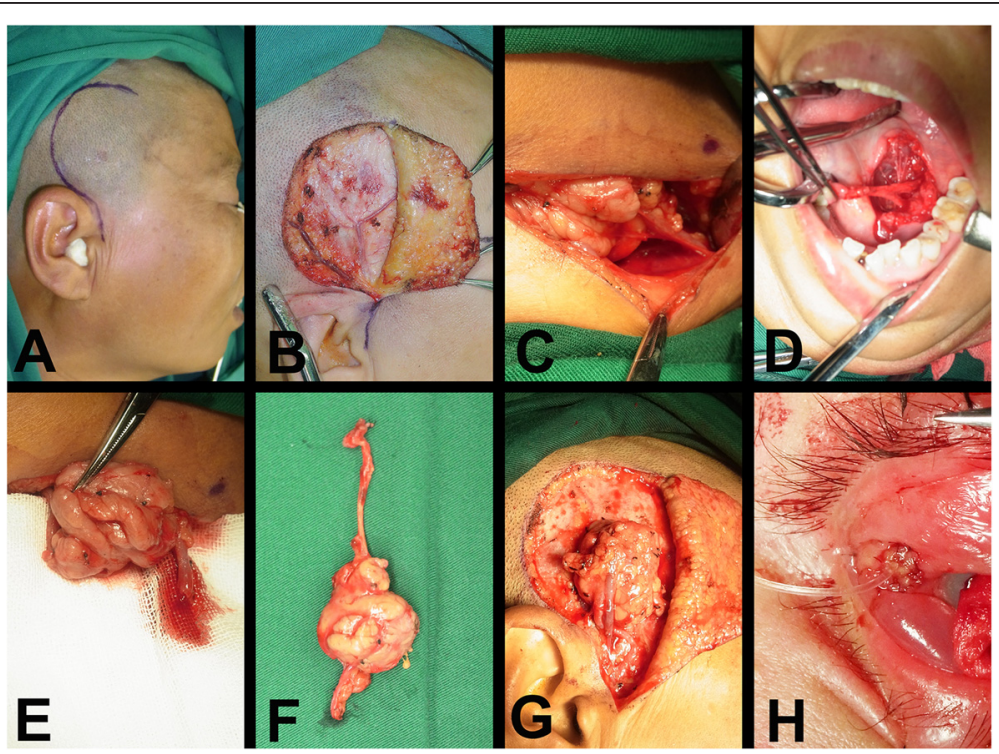

Figure 1 Surgical procedures of autologous microvascular submandibular gland transplantation. (A) Incision in the temporal region. (B) Dissection of the superficial temporal vessels. (C) Dissection of the facial artery and its accompanying vein. (D) Dissection of the Wharton's duct and a cuff of mucosa around the opening. (E) Bleeding from the anterior facial vein which attached to the submandibular gland was observed to evaluate the state of venous drainage from the gland. (F) The submandibular gland was removed together with the facial vessels and Wharton's duct. (G) Anastomosis of the vessels. (H) A nylon tube was inserted into the duct. 
vessels were cut and prepared for anastomosis. The superficial temporal artery was anastomosed with the facial artery by means of a 8-0 suture. The superficial temporal vein was anastomosed with anterior facial vein or the accompanying vein of the facial artery (Figure 1G).

Wharton's duct was passed through a tunnel prepared subcutaneously to the upper lateral conjunctival fornix by blunt dissection. The distal end of Wharton's duct was sutured to form an opening in the upper lateral conjunctival fold. A nylon tube was inserted into the duct and fixed on the skin of the lateral wall of the orbit (Figure 1H) $[10,18,19]$.

\section{Postoperative function regularity and ophthalmologic assessment}

In the patients with successful transplantation, the secretion of the transplanted glands underwent a regular change after transplantation. It could be divided into four stages: transient hypofunction period (1-2 days after operation), temporary epiphora period (3-6 days after operation), latent period (1 week-3 months after operation) and function recovery and stable period (more than 3 months after operation) $[10,20]$.

In the first 1-2 days after operation, very little secretion appeared. Then hypersecretion occurred in the "temporary epiphora period" (a mean value of $25 \mathrm{~mm}$ with Schirmer test). Secretion declined gradually over the next 3 months in "latent period" (mean value of Schirmer test, $5 \mathrm{~mm}$ ). At last the secretion rate picked up again and remained stable after 3 months (mean value of Schirmer test, $17 \mathrm{~mm}$ ) $[10,20]$.

Postoperative ${ }^{99 \mathrm{~m}}$ Tc-pertechnetate examination showed that the secretory function from all viable transplanted SMGs maintained stable function in the long term [21]. The lubricant effect of saliva appeared satisfactory with disappearance or relief of symptoms resulting from dry eye. The patients were able to eventually stop the application of artificial tear substitutes. Objective examination showed significant improvement in tear film and some features of ocular surface such as break-up time of tear film and corneal staining $[10,13,15]$.

\section{Postoperative complications \\ Duct obstruction}

The nylon tube inserted into the duct was kept for about 10 days, to prevent scar formation between the orifice of the duct and the conjunctiva and stricture of the duct opening. In some cases in which the orifices of the left and right Wharton's ducts were very close to each other, harvesting of the mucosal cuff was abandoned in order to avoid injury to the contralateral ductal orifice. In these circumstances, Wharton's duct had a naked end, and the ductal wall was directly sutured to the palpebral conjunctiva. For those patients a polyethylene tube would be intubated and left in the Wharton's duct for more than 1 month.
The transplanted gland is completely disconnected from normal nerve supply during surgery. As a result, the gland is hyposecretory for about 3 months after transplantation. During this latent period, transplanted SMGs are at a high risk of duct obstruction, a complication that results in insufficient ocular lubrication of the treated eyes, greatly impairs the effect of transplantation, and may even lead to treatment failure. The incidence of this complication was about $9.3 \%$ in the early phase (0-3 months) $[6,10,22]$. The chronic inflammation secondary to ductal obstruction (obstructive sialadenitis) of the transplanted SMG showed very low secretion and recurrent swelling fo the gland. Viscous secretions were expressed upon milking the swollen transplanted glands [22].

Promotion of secretion from the transplanted SMG during the latent period is the main point for prevention of ductal obstruction. Secretions from normal SMGs are primarily evoked by the action of acetylcholine on muscarinic receptors and adrenergic agonists on adrenoceptors [23]. In our systemic studies on secretion of SMG, a functional expression of transient receptor potential vanilloid subtype 1 (TRPV1) was found in human and rabbit SMGs [24,25]. In the rabbit models of experimental transplanted SMG, carbachol (an agonist of muscarinic receptors) and capsaicin (an agonist of TRPV1) improved secretory function of transplanted SMG in the early stage after transplantation $[25,26]$.

Clinically we found that combined administration of capsaicin and carbachol during the latent period could significantly increase the secretion of transplanted SMG and decrease the incidence of ductal obstruction [27]. As for the patients with duct obstruction of the transplanted SMG, reconstruction of the duct with venous grat or recontouring of the duct orifice would be the choice of treatment [10].

\section{Epiphora}

Secretion of the transplanted SMG is much more than that of lacrimal glands. Epiphora may occur in more than $40 \%$ of the patients with total SMG transplantation, especially in those with ample SMGs and normal function before surgery $[10,21]$. Severe epiphora may lead to social embarrassment and blurred vision. This probem is usually provoked by high room temperature or physical activity $[4,28]$, It is usually treated by gland reduction surgery, that is, removing approximately one third to half of the transplanted SMG to decrease the glandular secretion. However, the patients should undergo additional surgery after transplantation. According to our experience, transplantation of partial SMG can reduce the incidence of severe postoperative epiphora for the patients with ample SMGs and normal function [12]. For the patients with mild or moderate epiphora, satisfactory treatment effect of topical application of atropine gel could be achieved [25]. While 
reduction surgery is used for the patients with severe epiphora. Botulinum toxin A is also used for control of over-secretion of the transplanted SMG $[29,30]$.

\section{Conclusion}

Microvascular SMG transfer is effective for the severe cases of KCS. A comprehensive evaluation on the eyes and gland is needed before surgery. The Wharton's duct and the related blood vessels of SMG should be harvested intactly in the surgery. Venous drainage from the gland should be evaluated to choice the proper vein for anastomosis. Patients may suffer from obstruction of Wharton's duct or epiphora after surgery. Activation of secretion-related receptors could improve the early hypofunction of the denervated SMG and prevent the duct obstruction. Reduction surgery, partial SMG transplantation, uses of atropine gel or Botulinum toxin A could be the choices of treatment for epiphora.

\section{Competing interests}

The authors declare that they have no competing interest.

\section{Authors' contributions}

JZS, ZGC and GYY reviewed the references. JZS prepared the manuscript. ZGC and GYY revised critically for important intellectual content. All authors read and approved the final manuscript.

Received: 12 January 2015 Accepted: 12 January 2015

Published online: 05 February 2015

\section{References}

1. Lemp MA, Baudouin C, Baum J, Dogru M, Foulks GN, Kinoshita S, Laibson P, McCulley J, Murube J, Pflugfelder SC, Rolando, Toda I (2007) The definition and classification of dry eye disease: report of the definition and classification subcommittee of the international dry eye workShop (2007). Ocul Surf 5:75-92

2. Liu D, Sadhan Y (2002) Surgical punctal occlusion: a prospective study. Br J Ophthalmol 86:1031-1034

3. Farris RL (1991) Staged therapy for the dry eye. CLAO J 17:207-215

4. Geerling G, Sieg P, Bastian GO, Laqua H (1998) Transplantation of the autologous submandibular gland for most severe cases of keratoconjunctivitis sicca. Ophthalmology 105:327-335

5. Murube-del-Castillo J (1986) Transplantation of salivary gland to the lacrimal basin. Scand J Rheumatol Suppl 61:264-267

6. MacLeod AM, Robbins SP (1992) Submandibular gland transfer in the correction of dry eye. Aust N Z J Ophthalmol 20:99-103

7. Kumar PA, Hickey MJ, Gurusinghe CJ, O'Brien BM (1991) Long term results of submandibular gland transfer for the management of xerophthalmia. Br J Plast Surg 44:506-508

8. Sieg P, Geerling G, Kosmehl H, Lauer I, Warnecke K, von Domarus H (2000) Microvascular submandibular gland transfer for severe cases of keratoconjunctivitis sicca. Plast Reconstr Surg 106:554-560, discussion $561-552$

9. Jia G, Wang Y, Lu L, Wang X, Li Z (1998) Reconstructive lacrimal gland with free submandibular gland transfer for management of xerophthalmia. Zhonghua Yan Ke Za Zhi 34:388-390

10. Yu GY, Zhu ZH, Mao C, Cai ZG, Zou LH, Lu L, Zhang L, Peng X, Li N, Huang Z (2004) Microvascular autologous submandibular gland transfer in severe cases of keratoconjunctivitis sicca. Int J Oral Maxillofac Surg 33:235-239

11. Paniello RC (2007) Submandibular gland transfer for severe xerophthalmia. Laryngoscope 117:40-44

12. Qin J, Zhang L, Cai ZG, Mao C, Liu XJ, Lv L, Zou LH, Peng X, Su JZ, Wu J, Yu GY (2013) Microvascular autologous transplantation of partial submandibular gland for severe keratoconjunctivitis sicca. Br J Ophthalmol 97:1123-1128
13. Borrelli M, Schroder C, Dart JK, Collin JR, Sieg P, Cree IA, Matheson MA, Tiffany JM, Proctor G, van Best J, Hyde N, Geerling G (2010) Long-term follow-up after submandibular gland transplantation in severe dry eyes secondary to cicatrizing conjunctivitis. Am J Ophthalmol 150:894-904

14. Jacobsen HC, Hakim SG, Lauer I, Dendorfer A, Wedel T, Sieg P (2008) Long-term results of autologous submandibular gland transfer for the surgical treatment of severe keratoconjunctivitis sicca. J Craniomaxillofac Surg 36:227-233

15. Schroder C, Hakim SG, Collin JR, Sieg P, Geerling G (2003) Long-term follow-up after autologous submandibular gland transplantation in scarring keratoconjunctivitis with absolute dry eyes. Ophthalmologe 100:1079-1084

16. Schroder C, Sieg P, Framme C, Honnicke K, Hakim SG, Geerling G (2002) Transplantation of the submandibular gland in absolute dry eyes. Effect on the ocular surface. Klin Monbl Augenheilkd 219:494-501

17. Yu GY, Wu LL, Liu XJ (2009) Microvascular autologous submandibular gland transfer in severe cases of keratoconjunctivitis sicca: a 10-year experience. Chin J Dent Res 12:79-82

18. Zhang L, Yu GY (2013) Autotransplantation of submandibular gland for severe keratoconjunctivitis. In: McGurk M, Combes J (eds) Controversies in the management of salivary gland disease, 2nd edn. Oxford University Press, Oxford, pp 332-337

19. Yu GY, Ding C, Li YM, Wu LL (2013) Replacing tears with saliva: hope for patients with severe dry eye syndrome. In: Braxton L, Quinn S (eds) Salivary Glands: Anatomy, Functions in Digestion and Role in Disease (human anatomy and physiology: physiology-laboratory and clinical research). Nova Science Publisher, Inc, New York, pp 179-188

20. Zang L, Yu GY, Mao C, Cai ZG, Peng X, Huang Z, Zhu ZH, Lv L (2003) The secretion changes of transferred gland after microvascular autologous submandibular gland transfer and intervention. CJOMS 1:67-69

21. Geerling G, Garrett JR, Paterson KL, Sieg P, Collin JR, Carpenter GH, Hakim SG, Lauer I, Proctor GB (2008) Innervation and secretory function of transplanted human submandibular salivary glands. Transplantation 85:135-140

22. Su JZ, Yang NY, Liu XJ, Cai ZG, Lv L, Zhang L, Wu LL, Liu DG, Ren WG, Gao Y, Yu GY (2014) Obstructive sialadenitis of a transplanted submandibular gland: chronic inflammation secondary to ductal obstruction. Br J Ophthalmol 98:1672-1677

23. Proctor GB, Carpenter GH (2007) Regulation of salivary gland function by autonomic nerves. Auton Neurosci 133:3-18

24. Ding QW, Zhang Y, Wang Y, Wang YN, Zhang L, Ding C, Wu LL, Yu GY (2010) Functional vanilloid receptor-1 in human submandibular glands. J Dent Res 89:711-716

25. Zhang Y, Cong X, Shi L, Xiang B, Li YM, Ding QW, Ding C, Wu LL, Yu GY (2010) Activation of transient receptor potential vanilloid subtype 1 increases secretion of the hypofunctional, transplanted submandibular gland. Am J Physiol Gastrointest Liver Physiol 299:G54-G62

26. Shi L, Cong X, Zhang Y, Ding C, Ding QW, Fu FY, Wu LL, Yu GY (2010) Carbachol improves secretion in the early phase after rabbit submandibular gland transplantation. Oral Dis 16:351-359

27. Wang Y, Zhang L, Yu GY (2007) The effect of capsaicin on the secretion of the transferred submandibular gland in "latent period". Chin J Stomatol 42:161-164

28. Su JZ, Liu XJ, Zhang L, Yu GY (2013) Schirmer test in transplanted submandibular gland: influencing factors and a modified measurement method. Cornea 32:419-422

29. Keegan DJ, Geerling G, Lee JP, Blake G, Collin JR, Plant GT (2002) Botulinum toxin treatment for hyperlacrimation secondary to aberrant regenerated seventh nerve palsy or salivary gland transplantation. $\mathrm{Br} J$ Ophthalmol 86:43-46

30. Shan XF, Xu H, Cai ZG, Wu LL, Yu GY (2013) Botulinum toxin A inhibits salivary secretion of rabbit submandibular gland. Int J Oral Sci 5:217-223 